\title{
Association of a Giant Lymphoma Mass With Occupational Benzene Exposure
}

\author{
Yota Suzukia $^{\text {a }}$ Shawn Robinson ${ }^{\mathrm{b}}$, Ikenna Okereke ${ }^{\mathrm{b}, \mathrm{c}}$
}

\begin{abstract}
Benzene is a chemical widely used in industrial settings and known to have hematopoietic toxicity. Previous literature has been inconsistent regarding a direct association of benzene exposure with lymphoma. A young patient with a strong history of benzene exposure presented with a $17-\mathrm{cm}$ chest wall lymphoma and purulent drainage from the mass. He underwent resection of the mass. Pathologic analysis revealed a high-grade diffuse large B-cell lymphoma. The wound ultimately healed well and the patient received adjuvant chemotherapy. Though the overall literature has been inconsistent regarding the association of benzene with lymphoma, our report highlights the possibility that a very extensive exposure history may be a risk factor for the development of lymphoma.
\end{abstract}

Keywords: Lymphoma; Chest wall tumor; Benzene; Carcinogen

\section{Introduction}

Benzene is a known carcinogen and has been shown to be a risk factor for many hematopoietic diseases such as leukemia. The association of benzene with lymphoma is less clear, however. We report a case of a patient with a giant chest wall lymphoma, diffuse disease and a strong history of benzene exposure.

\section{Case Report}

A 45-year-old previously healthy man presented to our institution with a large mass in his left axilla. The mass had been present for several months but had grown significantly in re-

\footnotetext{
Manuscript submitted September 2, 2019, accepted September 9, 2019

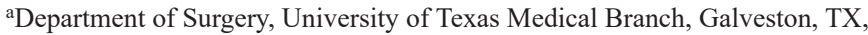
USA

bivision of Cardiothoracic Surgery, University of Texas Medical Branch, Galveston, TX, USA

${ }^{\mathrm{c} C}$ Corresponding Author: Ikenna Okereke, Division of Cardiothoracic Surgery, University of Texas Medical Branch, 301 University Blvd, Galveston, TX 77555, USA. Email: ikokerek@utmb.edu
}

cent weeks. The mass measured $17 \mathrm{~cm}$ in maximum dimension and was relatively mobile. The mass had developed superficial skin ulceration and was malodorous (Fig. 1). There was purulent material draining from the ulcerated areas. Of note, the patient was a factory worker in an industrial plant and had been exposed to benzene for over 20 years. A computed tomography (CT) scan of the chest revealed the large axillary mass, multiple bilateral pulmonary nodules and splenomegaly with rounded, hypodense areas (Fig. 2).

Given that the axillary mass was infected, the patient underwent a complete resection of the mass. The mass abutted the axillary vein and thoracodorsal nerve, but these structures were spared. The skin was kept open and had a wound vacuum dressing applied. The patient had an uneventful postoperative course and was discharged on day 4.

Histologic analysis of the mass showed a diffuse lymphoid infiltrate of predominantly large atypical lymphoid cells. Immunohistochemical staining was positive for cluster of differentiation 2 (CD2), CD79a, paired box-5 (PAX5), B-cell lymphoma-2 (BCL-2) and BCL-6. Staining was negative for CD10, CD5, CD43, CD34, multiple myeloma oncogene 1 (MUM-1) and terminal deoxynucleotidyl transferase (TdT) as well as myelocytomatosis oncogene (c-myc). Fluorescence in situ hybridization (FISH) was positive for both c-myc and BCL-6. With these results, he was diagnosed with a double-hit, high-grade B-cell lymphoma. The patient was given three cycles of including dose adjusted etoposide, prednisone, vincristine, cyclophosphamide, doxorubicin, and rituximab (DA-EPOCH-R). On follow-up in 7 months after

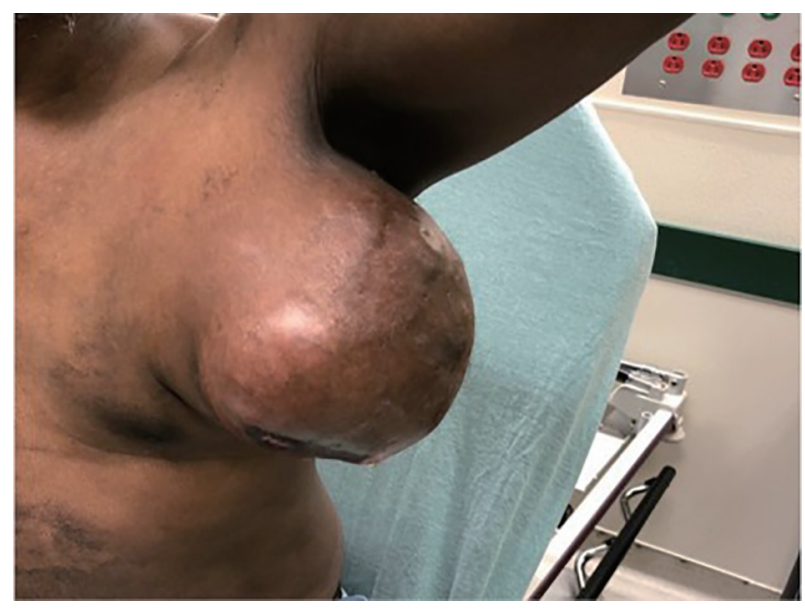

Figure 1. Large axillary double-hit lymphoma. 


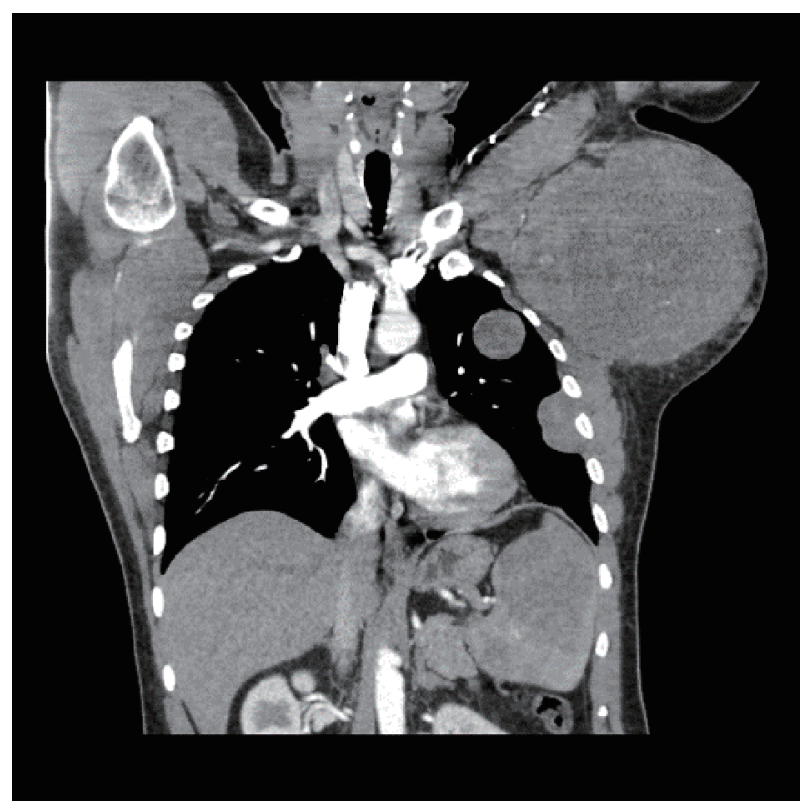

Figure 2. Contrast-enhanced computed tomography showing axillary mass, lung nodules and hypodense lesions in the spleen.

surgery, he was asymptomatic and the wound had healed completely.

\section{Discussion}

Benzene is an organic chemical compound which has historically been used as a component of inks, solvents for organic materials and as a gasoline. There have been conflicting accounts in the literature concerning the association of benzene with lymphoma. In a report by the International Agency for Research on Cancer in 2012, an increased risk of non-Hodgkin's lymphoma (NHL) secondary to benzene exposure was shown in five of 14 case-control studies [1]. Other metaanalyses have shown no clear connection, however [2]. While there is sufficient evidence to support a relationship between occupational benzene exposure and leukemia [3], the data supporting a connection between benzene and NHL have been equivocal.

Our case featured a patient with a history of benzene exposure for decades and a considerable lymphoma tumor burden, with the largest deposit measuring $17 \mathrm{~cm}$. Although the benzene exposure and lymphoma development may have been coincidental in this patient, the very prolonged exposure history and the substantial bulkiness of disease raise the possibility of a causal relationship. There is no method by which it can be proven that benzene causes lymphoma in any isolated case. But continued reports in the literature may bolster population studies to show an association, and as such we feel there is value in this report.

Our case is also unique in that it highlights a case of lymphoma which required initial surgical resection. Although most cases of B-cell lymphoma need surgery only when a diagnosis cannot otherwise be made, this patient had a giant, infected tumor deposit. Chemotherapy administration was not feasible without resection of the mass. Once the infected source was removed, however, he did quite well with systemic treatment and is in good functional condition now.

Our patient was diagnosed with a double-hit B-cell lymphoma. "Double-hit" and "triple hit" lymphomas are a relatively new designation, first advocated in the 2016 revision of the World Health Organization classification [4]. The diagnosis is determined by FISH to detect c-myc, BCL2 and/or BCL6 rearrangements [5]. The incidence of double-hit or triple-hit rearrangements is approximately $5 \%$ of all diffuse large Bcell lymphomas. When found, patients are more likely to have extranodal disease, central nervous system involvement and worse prognosis [6]. Although there has been no study showing a dose-related association of benzene with double-hits and triple-hits, theoretically an increased exposure to the offending agent could lead to a more severe form of the disease. This hypothesis could explain our case of a high-risk lymphoma with substantial tumor burden in a relatively young patient, as he had been exposed to so much benzene in his life.

\section{Acknowledgments}

None to declare.

\section{Financial Disclosure}

Departmental sources funded this project.

\section{Conflict of Interest}

None to declare.

\section{Informed Consent}

Consent was obtained to publish this report.

\section{Author Contributions}

YS, SR and IO contributed to the writing of the manuscript. IO supervised all aspects of the manuscript.

\section{References}

1. Loomis D, Guyton KZ, Grosse Y, El Ghissassi F, Bouvard V, Benbrahim-Tallaa L, Guha N, et al. Carcinogenicity of benzene. Lancet Oncol. 2017;18(12):1574-1575.

2. Kane EV, Newton R. Benzene and the risk of non-Hodgkin lymphoma: a review and meta-analysis of the literature. Cancer Epidemiol. 2010;34(1):7-12.

3. Snyder R. Leukemia and benzene. Int J Environ Res Public Health. 2012;9(8):2875-2893. 
4. Swerdlow SH, Campo E, Pileri SA, Harris NL, Stein $\mathrm{H}$, Siebert R, Advani R, et al. The 2016 revision of the World Health Organization classification of lymphoid neoplasms. Blood. 2016;127(20):2375-2390.

5. Rosenthal A, Younes A. High grade B-cell lymphoma with rearrangements of MYC and BCL2 and/or BCL6:
Double hit and triple hit lymphomas and double expressing lymphoma. Blood Rev. 2017;31(2):37-42.

6. Landsburg DJ, Falkiewicz MK, Maly J, Blum KA, Howlett C, Feldman T, Mato AR, et al. Outcomes of patients with double-hit lymphoma who achieve first complete remission. J Clin Oncol. 2017;35(20):2260-2267. 